\title{
Disturbances in the US electric grid associated with geomagnetic activity
}

\author{
Carolus J. Schrijver* and Sarah D. Mitchell \\ Lockheed Martin Advanced Technology Center, Palo Alto, CA, USA \\ *Corresponding author: e-mail: schrijver@lmsal.com
}

Received 8 November 2012 / Accepted 19 April 2013

\begin{abstract}
Large solar explosions are responsible for space weather that can impact technological infrastructure on and around Earth. Here, we apply a retrospective cohort exposure analysis to quantify the impacts of geomagnetic activity on the US electric power grid for the period from 1992 through 2010 . We find, with more than $3 \sigma$ significance, that approximately $4 \%$ of the disturbances in the US power grid reported to the US Department of Energy are attributable to strong geomagnetic activity and its associated geomagnetically induced currents.
\end{abstract}

Key words. solar magnetic activity - geomagnetic disturbances - US electric power grid - geomagnetically induced currents

\section{Introduction}

Explosions powered by the Sun's magnetic field ("flares" and "coronal mass ejections" or CMEs) are among the principal causes of "space weather" (see, e.g., Space Studies Board 2008). These electromagnetic storms can affect our technological infrastructure in space, interfere with communications and GPS signals, and couple through the geomagnetically induced currents (GICs) into the large-scale high-voltage electric grid (see, e.g., Boteler et al. 1998; Boteler \& Jansen van Beek 1999; Gaunt \& Coetzee 2007). Despite the known impact of large space weather events on the electrical power grid (see, e.g., Space Studies Board 2008; FEMA 2010; Kappenman 2010; Hapgood 2011; JASON 2011) - including the 1989 Hydro-Québec blackout (Béland \& Small 2004) - relatively few studies of the general correlation are available; case studies of individual events (such as by Kappenman et al. 1997; Kappenman 2005) and compilations of events for comparison with the solar cycle (e.g., by Boteler et al. 1998) generally focus on large storms and large impacts.

There is a recognized hazard of catastrophic outages that may be caused by geomagnetic superstorms larger than what we have experienced in recent decades (Space Studies Board 2008; FEMA 2010; Kappenman 2010; Hapgood 2012). Such superstorms may cause trillions of dollars of damage (Space Studies Board 2008), although it is acknowledged that such estimates are rather uncertain (JASON 2011). Other studies assessing the economic impact on a statistical basis find significant correlations between magnetometer data, GICs, electric grid effects, and the conditions of the electric power grid market (Forbes \& St. Cyr 2004, 2008, 2010). These correlations are associated with market price variations on the order of a few percent (Forbes \& St. Cyr 2004).

The main cause of GICs is the interaction of the geomagnetic field with the magnetic field carried within CMEs and the surrounding background magnetized solar wind that is modulated by them. With speeds of $400-2500 \mathrm{~km} / \mathrm{s}$, it takes some
1-4 d for CMEs to propagate from the Sun to the Earth, with a typical transit time of 2-3 d. Correlations between the strength of CMEs, and the magnitude of their impact in geospace continue to be studied, both observationally and in numerical analyses (e.g., Newell et al. 2007; Schrijver 2009; Andreeova et al. 2011). A multitude of factors may play a role, including properties of the solar events themselves and of the solar wind through which the events travel to Earth (e.g., Russell \& McPherron 1973; Pulkkinen 2007; Schrijver \& Siscoe 2010). Furthermore, the magnitude of GICs depends on the location and time of day (through the geomagnetic position relative to the Sun-Earth line) at impact, on the structure of the magnetic field within the CME as that field interacts with the magnetic field of the Earth during the CME's passage (thereby inducing electric fields in the Earth dependent on the direction and the rate of change of the CME magnetic field), on the ground conductivities in a wide area around any particular site for depths from ground level down to in excess of $100 \mathrm{~km}$, and on the evolving architecture of the electric power grid into which the induced electric field couples.

Within the electric power system, GICs can cause transformers to operate in their nonlinear saturation range during half of the AC cycle. The consequences of half-cycle saturation include distortions of the voltage pattern (reflected in the existence of harmonics to the primary frequency), heating within the transformers, or voltage-to-current phase shifts expressed as reactive power consumption in the system. The detection of harmonics or of strong GICs may cause protective systems to trip, taking one or more transformers off line to protect them from severe damage. The implementation of such protective measures changes the grid's overall configuration as well as the regional balance between power generation and use which, in turn, can lead to power-quality variations in the form of voltage and frequency swings (causing, for example, the 1989 Hydro-Québec blackout, e.g., Boteler 2001). Moreover, the detection of GICs may cause system operators to change the operational standards to protect the overall system from 
damage, for example by changing the transfer limits for power that may be transported between segments of a grid (from where surplus power is more economically available to regions where the demand is highest) to create a buffer interval to keep GICs from pushing transformers into their nonlinear range (as is the standard "GMD procedure" during strong GIC events for the PJM regional transmission operator on the east coast of the US, see PJM State and Member Training Dept. 2010). Strong GICs can result in dissipative heating within the transformers which may lead to their failure, either within minutes or because of cumulative damage done over the lifetime of the transformer.

The strengths of GICs scale with the rate of change of the geomagnetic field. As our study addresses the reliability of the US power grid, we chose to use a measure of geomagnetic variability derived from geomagnetic measurements made around the central latitudes of the US. We verified that the use of a commonly used metric for large-scale geomagnetic variability, the $K p$ index, yields the same results when allowing for the statistical uncertainties. We find that even using criteria based directly on the occurrence of the solar events that ultimately drive space weather yields the same results, so that our findings are quite insensitive to the metric used to quantify space weather conditions in which the US power grid operates.

\section{Disturbances in the US power grid}

As input to this study we use a compilation of "system disturbances" published annually by both the North American Electric Reliability Corporation (NERC ${ }^{1}$; available since 1992) and by the Office of Electricity Delivery and Energy Reliability of the Department of Energy (DOE ${ }^{2}$; available since 2000). NERC compiles this information for an electric power market that serves over 300 million people throughout the USA and in Ontario and New Brunswick in Canada, jointly delivering power through more than $340,000 \mathrm{~km}$ of high-voltage transmission lines, linking 18,000 power plants within the US (JASON 2011).

The reported disturbances include, among others, "electric service interruptions, voltage reductions, acts of sabotage, unusual occurrences that can affect the reliability of the bulk electric systems, and fuel problems." The NERC reporting changed from "selected disturbances" to a more comprehensive listing starting in 2003 (following a grid collapse on August 14, 2003, affecting almost 50 million customers). The DOE lists add information for 2008,2010 , and 2011. To avoid a strongly inhomogeneous data set, we exclude the DOE data for 2011 because of a marked change in the types of events being reported on; for example, there are 79 events marked "Vandalism" in 2011, which is $300 \times$ the average rate for that class of event reported in the 19 preceding years. We thus use the combined disturbance reports ${ }^{3,4}$ for the 19-y period of 1992 through 2010.

We extracted the information on all 1,216 disturbances listed in the NERC-DOE reports, including the identified main cause, and the impact on power and number of customers affected (the latter two are often incompletely specified in the

\footnotetext{
${ }^{1} \mathrm{http}: / /$ www.nerc.com

${ }^{2}$ http://energy.gov/oe/office-electricity-delivery-and-energyreliability

${ }^{3}$ http://www.nerc.com/page.php?cid=5|66

4 http://www.oe.netl.doe.gov/OE417_annual_summary.aspx
}

disturbance reports). Hence, our master list of attributed "causes" includes a variety of weather conditions (storms, ice, lightning, etc.), operator errors, equipment failures, transmission line faults, etc.

Figure 1 shows that the overall frequency of grid disturbances exhibits a long-term increasing trend (the gray dashed curve), modulated substantially on shorter time scales (shown on a monthly basis by the black histogram). The figure also shows the yearly averaged sunspot number (green curve) that is - as expected - clearly correlated with flare frequency (blue line; shown inverted simply to avoid too much overlap with the grid-disturbance frequency). No obvious correlation between solar flaring activity and grid-disturbance frequency stands out (the peaks in the blue and black curves do not align, nor do the dotted vertical lines - dates of the most severe solar activity with at least two X-class flares - point to particularly enhanced griddisturbance frequencies), consistent with our conclusions below that the effects are relatively weak, albeit significant.

\section{Geomagnetic activity and electric power grid disturbances}

As no direct attributions to space-weather conditions have been made for the events from the NERC-DOE reports studied here, we anticipate at most a weak effect by space weather on the power grid that may be strongly modulated by other processes affecting the grid's condition. Given enough independent controlling variables (such as the evolving connectivities within the power grid, the patterns of weather conditions, and the grid loads and their changes with time around the country), one might develop a multivariate dependent variable model. However, insufficient information is available to us at present: the detailed supply, demand, and weather conditions are not included in the NERCDOE reports, and no information is available on the probability that no reportable grid disturbances ensued from other operator errors, cases of vandalism, or cyber attacks, for example. Moreover, as we find below that only a few dozen disturbances in the sample of over 1,000 reported disturbances are attributable to enhanced space weather, we cannot study separate grid areas while maintaining statistical significance of the results. Such regional studies are natural follow-up studies of this work, and those can focus not on the statistical demonstration of susceptibility, as we do here, but on the detailed physics of the electromagnetic coupling of GICs into the power grid.

The power grid is generally operated in a state with enough power being generated to meet customer demand, with only a relatively small overcapacity - the "reserve margin" - available to accommodate for rapid changes in demand or to compensate for "contingency events", that is, external perturbations of the grid, such as lightning strikes and other weather conditions, or internal events, such as component failures. Thus, whereas one might argue that, for example, disturbances attributed to a lightning strike or to an ice storm or to a heat wave might need to be removed from the list of disturbances in a study looking to quantify the potential effects of space weather, it may well be that the grid disturbance ensued only because other factors, possibly including space weather, put the system in a state of increased susceptibility. Taking this perspective, the only disturbances that one might exclude a priori are those that are attributed to planned maintenance (provided these did not cause unforeseen disturbances elsewhere) or to fuel shortages at the generating plants. Even cases flagged as "operator errors" should not be excluded a priori because the reports do not 


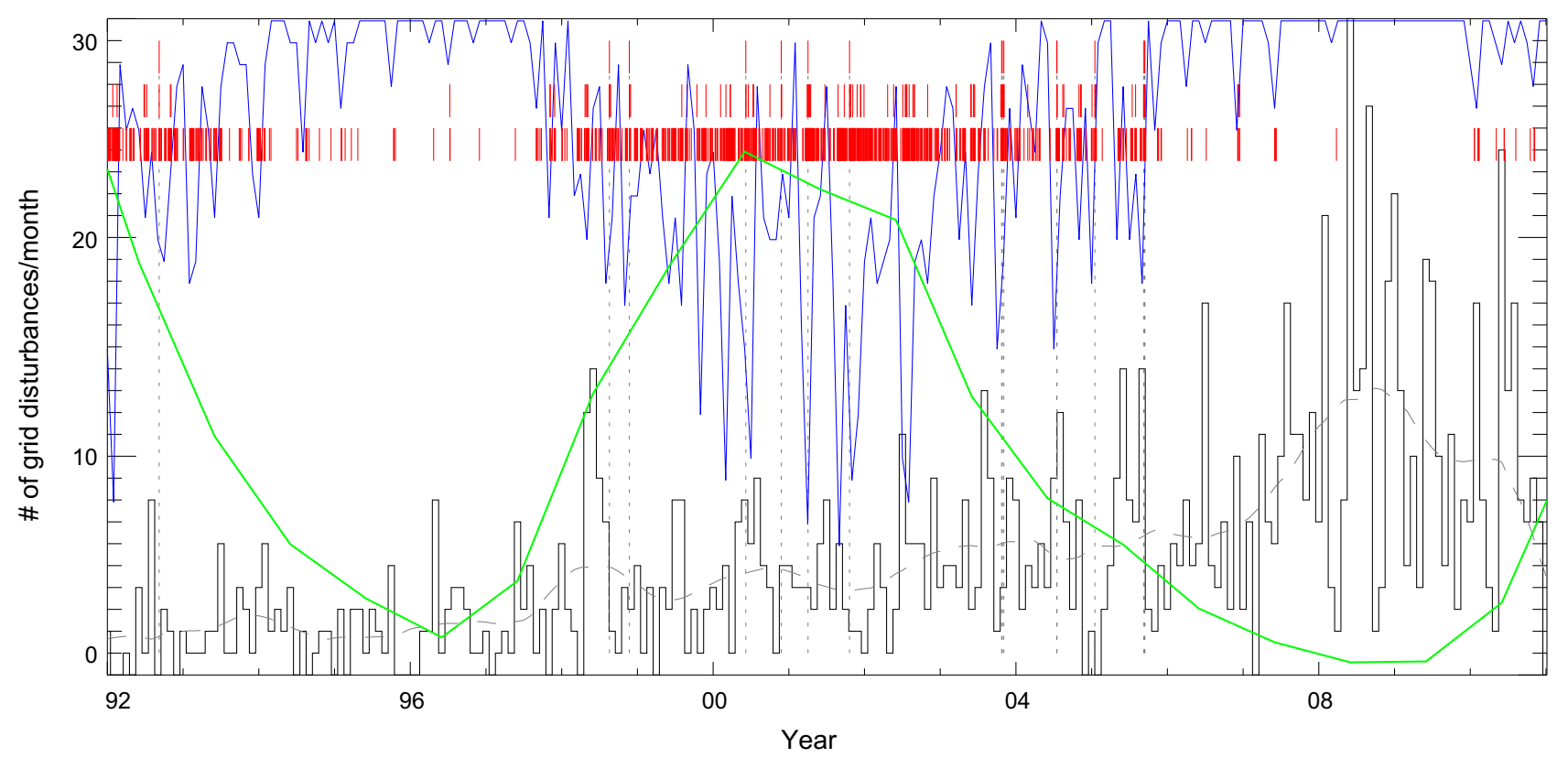

Fig. 1. Monthly frequency of grid disturbances (black histogram) and its 12-month running average (dashed gray line). Dates with (a) one of more M- or X-class flares, (b) one or more X-class flares, or (c) two or more X-class flares are marked by (red) bars near the top of the diagram. The monthly count of M- and X-class flares is shown inverted from the top by the blue line only to avoid confusing overlaps between this and the rate of grid disturbances, while still being able to view the relative behavior in the peaks of each of the two curves. The yearly sunspot number is overlaid as a (green) solid curve (divided by a factor of 5 for display purposes).

specify if the operators were responding to changing grid conditions or merely to a truly local need to change the operation of a grid segment. Even "vandalism" might be more or less effective in causing a grid disturbance depending on system load and on the conditions of the geomagnetic field. In view of the low numbers of events in the above sets, and as we do not wish to inadvertently introduce biases in the process, we elected to work with the complete set of reported grid disturbances.

Our study thus applies a standard method as used in, for example, epidemiology where it would be described as as a retrospective cohort exposure study with tightly matched controls (see, e.g., Schulz \& Grimes 2002, on cohort vs. case-control studies). In our case, the cohort under study is the set of all dates from 1992 through 2010. For all elements of that set, we study the "exposure" of the US electric power grid to geomagnetic activity in excess of a specified threshold (in fact, three distinct thresholds based on percentiles of the distribution of geomagnetic-activity values, as defined below) and count the number of power-grid disturbances on such dates. The results of that are then compared to two control samples with distinctly different levels of "exposure," namely one with average exposure levels and another - the reference control sample - with low exposure levels.

As the grid, its load, and its operating procedures change over time, we apply a method that compares grid-disturbance frequencies on days of elevated geomagnetic activity to a control sample of days of low geomagnetic activity but with all other conditions being similar. We control against effects of continuously varying confounders that are associated with the evolution of the grid's infrastructure and operating rules over time by sample matching, specifically by ensuring time comparability of the "exposed" and control samples (e.g., Wacholder et al. 1992, and references therein): we form two control samples with matched frequencies by selecting dates near each of the dates of high "exposure" subject only to a criterion about their exposure levels. The selection of two control samples, rather than only one, provides additional insight into the effects at three different levels of exposure that can be compared within each selected exposure percentile, but we caution against comparison across percentiles because of the changes in grid operating conditions with time.

In the definition of our control samples, we assume that weather conditions, fuel prices, and vandalism, for example, are not correlated with conditions on the Sun and in geospace within the 50-day sample matching windows (described below), but that these and other conditions form a background that varies independently of solar and space weather. In view of the above, we adopt the following avenue of research: we compare the frequency of grid disturbances under severe spaceweather conditions with that under light space-weather conditions, with the grid in otherwise similar conditions. The second group is the control group containing grid disturbances that are much less, if not entirely unaffected by space weather. The contrast between these two samples enables us to estimate the attributable risk, that is, the impact of geomagnetic disturbances associated with space weather.

To characterize the geomagnetic activity that may couple into the US power grid, we use data from the Boulder (BOU) and Fredericksburg (FRD) stations. ${ }^{5}$ With the minute-by-minute data in hand, we compute the maximum value of $|\mathrm{d} B / \mathrm{d} t|$ for 30 -min intervals, for the average of the two stations that are located along the central latitudinal axis of the US, somewhat emphasizing the eastern US as do the grid and population.

In Table 1, we list the average grid-disturbance rates, $g_{a}$, for dates corresponding to the top $p=2,5$, and 10 percentiles of geomagnetic activity, respectively. These numbers need to be compared to disturbance rates in the absence of strong geomagnetic activity. In order to ensure that the grid and its load are in

\footnotetext{
${ }^{5}$ http://ottawa.intermagnet.org/apps/dl_data_def_e.php
} 
Table 1. Average daily frequency of grid disturbances for three distinct selection criteria: $g_{a}$ within the day of high geomagnetic activity as measured by $|\mathrm{d} B / \mathrm{d} t|(30 \mathrm{~m}) ; g_{i}$ for a day ending a 3-day period with the lowest average $|\mathrm{d} B / \mathrm{d} t(30 \mathrm{~m})|$ within 25 days of a day with high $\mid \mathrm{d} B /$ $\mathrm{d} t(30 \mathrm{~m}) \mid ; g_{r}$ for a day selected at random between 5 and 50 days before or after high $|\mathrm{d} B / \mathrm{d} t(30 \mathrm{~m})|$. The conditional criterion for days with high $|\mathrm{d} B / \mathrm{d} t(30 \mathrm{~m})|$ is defined in the first column for each of the three rows. The final column shows the total number of dates, $N_{d}$ with high $\mid \mathrm{d} B /$ $\mathrm{d} t(30 \mathrm{~m}) \mid$ corresponding to the 2,5 , and 10 percentile levels. Uncertainties in $f_{a}$ and $f_{i}$ assume Poisson statistics; for $f_{r}$ the standard deviation of a sample of 100 random realizations is given. Data are shown for all grid disturbances (top), for grid disturbances attributed to weather, technical or external causes (center), and for the complementary set of grid disturbances of unclear attribution (bottom).

\begin{tabular}{|c|c|c|c|c|}
\hline Selection criterion for reference dates & $g_{a}$ (enhanced geomagn. act) & $g_{i}$ (low nearby geomagn. act) & $g_{r}$ (nearby random date) & $N_{d}$ \\
\hline $\begin{array}{l}\text { All disturbances (1,216 cases }) \\
|\mathrm{d} B / \mathrm{d} t(30 \mathrm{~m})| \geq 36.1 \\
|\mathrm{~d} B / \mathrm{d} t(30 \mathrm{~m})| \geq 24.5 \\
|\mathrm{~d} B / \mathrm{d} t(30 \mathrm{~m})| \geq 18.5\end{array}$ & $\begin{array}{l}0.230 \pm 0.041 \\
0.184 \pm 0.023 \\
0.167 \pm 0.016\end{array}$ & $\begin{array}{l}0.058 \pm 0.020 \\
0.107 \pm 0.018 \\
0.089 \pm 0.012\end{array}$ & $\begin{array}{l}0.136 \pm 0.031 \\
0.143 \pm 0.023 \\
0.147 \pm 0.016\end{array}$ & $\begin{array}{l}139 \\
347 \\
694\end{array}$ \\
\hline $\begin{array}{l}\text { WET: Attributed to weather/external/t } \\
|\mathrm{d} B / \mathrm{d} t(30 \mathrm{~m})| \geq 36.1 \\
|\mathrm{~d} B / \mathrm{d} t(30 \mathrm{~m})| \geq 24.5 \\
|\mathrm{~d} B / \mathrm{d} t(30 \mathrm{~m})| \geq 18.5\end{array}$ & $\begin{array}{c}\text { anical causes }(743 \text { cases }) \\
0.137 \pm 0.031 \\
0.115 \pm 0.018 \\
0.099 \pm 0.011\end{array}$ & $\begin{array}{l}0.043 \pm 0.018 \\
0.055 \pm 0.013 \\
0.050 \pm 0.009\end{array}$ & $\begin{array}{l}0.070 \pm 0.024 \\
0.077 \pm 0.016 \\
0.080 \pm 0.010\end{array}$ & \\
\hline $\begin{array}{l}\text { U: Unclear/unknown attribution (473 } \\
|\mathrm{d} B / \mathrm{d} t(30 \mathrm{~m})| \geq 36.1 \\
|\mathrm{~d} B / \mathrm{d} t(30 \mathrm{~m})| \geq 24.5 \\
|\mathrm{~d} B / \mathrm{d} t(30 \mathrm{~m})| \geq 18.5\end{array}$ & $\begin{array}{l}0.094 \pm 0.026 \\
0.069 \pm 0.010 \\
0.068 \pm 0.010\end{array}$ & $\begin{array}{l}0.014 \pm 0.010 \\
0.052 \pm 0.012 \\
0.039 \pm 0.007\end{array}$ & $\begin{array}{l}0.066 \pm 0.025 \\
0.066 \pm 0.017 \\
0.068 \pm 0.013\end{array}$ & \\
\hline
\end{tabular}

Table 2. Average daily frequency of grid disturbances for three distinct selection criteria: $f_{a}$ from 2 to 5 days after a major flare; $f_{i}$ for inactive intervals, i.e., 4-d intervals following the first 7-d intervals of no $\mathrm{M}$ or X flaring prior to dates with major flaring; $f_{r}$ for a randomly-selected 4-d interval between 5 and 50 days before or after "major flaring". The conditional criterion for days with "major flaring" is defined in the first column for each of the three rows. The final two columns show the total number of dates, $N_{d}$, and the total number of flares on such dates, $N_{f}$. Uncertainties and subsampling criteria are as defined in Table 1.

\begin{tabular}{lcccc}
\hline \hline Selection criterion for reference dates & $\begin{array}{c}f_{a}(2-5 \mathrm{~d} \text { after } \\
\mathrm{M} / \mathrm{X} \text { flaring) }\end{array}$ & $\begin{array}{c}f_{i} \text { (nearby interval, } \\
\text { without M/X flaring) }\end{array}$ & $\begin{array}{c}f_{r} \text { (random } \\
\text { nearby date) }\end{array}$ & $N_{d}$ \\
\hline All disturbances (1,216 cases) & & & & \\
Multiple X flares & $0.328 \pm 0.072$ & $0.063 \pm 0.031$ & $0.210 \pm 0.071$ & 16 \\
At least one X flare & $0.179 \pm 0.020$ & $0.116 \pm 0.015$ & $0.154 \pm 0.022$ & 116 \\
At least one M or X flare & $0.151 \pm 0.006$ & $0.126 \pm 0.005$ & $0.148 \pm 0.007$ & 1,054 \\
WET: Attributed to weather/external/technical causes (743 cases) & & & \\
Multiple X flares & $0.140 \pm 0.047$ & $0.031 \pm 0.022$ & $0.120 \pm 0.056$ \\
At least one X flare & $0.071 \pm 0.012$ & $0.050 \pm 0.010$ & $0.085 \pm 0.017$ \\
At least one M or X flare & $0.077 \pm 0.004$ & $0.068 \pm 0.004$ & $0.083 \pm 0.005$ \\
U: Unclear/unknown attribution (473 cases) & & & & \\
Multiple X flares & $0.188 \pm 0.054$ & $0.031 \pm 0.022$ & $0.090 \pm 0.040$ \\
At least one X flare & $0.108 \pm 0.015$ & $0.067 \pm 0.012$ & $0.067 \pm 0.011$ & \\
At least one M or X flare & $0.074 \pm 0.004$ & $0.058 \pm 0.004$ & $0.064 \pm 0.004$ & \\
\hline
\end{tabular}

a statistically comparable state, we look at conditions in $50-\mathrm{d}$ windows centered on dates with high $|\mathrm{d} B / \mathrm{d} t(30 \mathrm{~m})|$. Selecting a random date within these windows, but more than $5 \mathrm{~d}$ away from the reference dates, yields the disturbance rates $g_{r}$. These are lower than the rates $g_{a}$ for days of high geomagnetic activity, but this selection criterion does not, of course, avoid dates of significant geomagnetic activity. Hence, for a second control sample we select dates for the last day of the 3-d interval of the lowest average $|\mathrm{d} B / \mathrm{d} t(30 \mathrm{~m})|$ within each of the 50 - $\mathrm{d}$ intervals. This yields disturbance rates $g_{i}$ for geomagnetically inactive days.

We note that for each of the percentile $(p)$ levels, we find $g_{a}>g_{r}>g_{i}$, that is, the disturbance frequency is highest within geomagnetically active days, lower for a randomly sampled nearby day, and lowest when geomagnetic activity is lowest. We caution that the values of $g_{a, r, i}$ for different $p$ levels are not directly comparable, because the coverage throughout the full sample period for each of these sets is different, and thus sensitive to long-term trends in grid, weather, and solar cycle.

For each value of the percentiles, $p$, we can estimate the number of grid disturbances in excess of those occurring in conditions of low geomagnetic activity by computing $N_{p}=\left(g_{a}-g_{i}\right)(p /$ $100) n_{d}$ (where $n_{d}$ is the number of days in our 19-y study interval): $N_{p}=24 \pm 6,27 \pm 10$, and $54 \pm 13$, respectively, for $p=2,5$, and 10. For higher $p$ values, more disturbances may be associated with geomagnetic activity, but the uncertainties on the values of $N_{d}$ rapidly increase (for $p=25$, for example, the uncertainty in $N_{25}$ embraces $N_{10}$ within one standard deviation), so that with the present data, we leave it at our finding that at least $N_{10} \approx 50$ disturbances are attributable to enhanced geomagnetic activity during the period of our study.

In order to assess whether our choice of metric for geomagnetic variability would significantly bias the results, we repeated our analysis for another commonly used index to characterize the interaction of the geomagnetic field with the variable solar 
C.J. Schrijver and S.D. Mitchell: Grid disturbances associated with geomagnetic activity
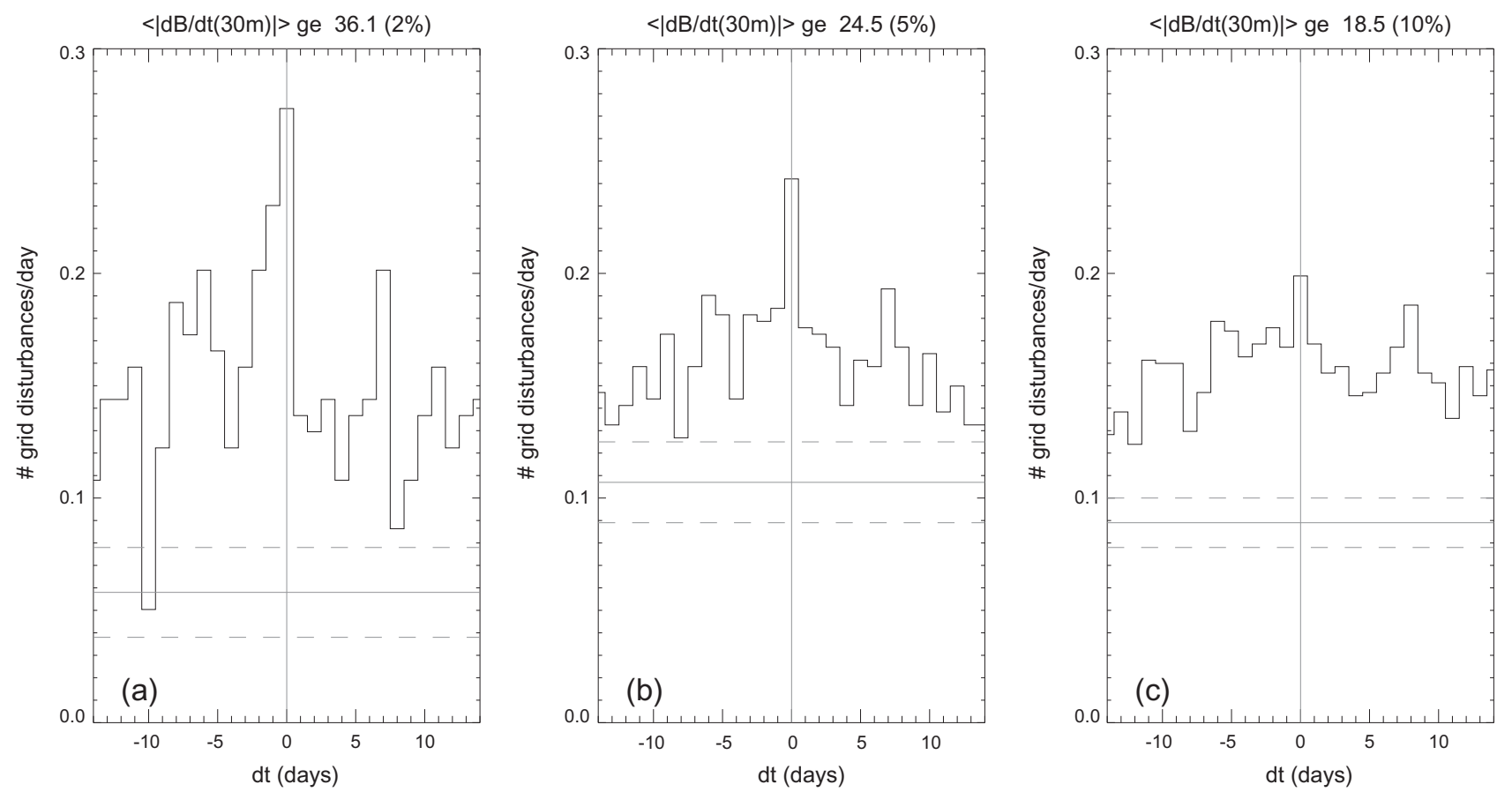

Fig. 2. Superposed epoch statistics of US power-grid disturbances for days of geomagnetic activity as measured by the maximum $\mathrm{d} B / \mathrm{d} t$ in 30-min intervals, averaged for the US BOU and FRD stations $(\langle|\mathrm{d} B / \mathrm{d} t(30 \mathrm{~m})|\rangle)$ for the top 2, 5, and $10 \%$ of dates between 1992 and 2010 , respectively. In each panel, the horizontal gray line and the dashed lines adjacent to it show the disturbance rates under quiescent space-weather conditions (i.e., the values of $g_{i}$, see Sect. 3 and Table 1) and the associated standard deviations, respectively.

wind, namely the $K p$ index. $K p$ is measured in sub-auroral midlatitude stations characteristic of activity in central regions of Europe and the northern US, which is to be contrasted to the higher latitudes used for the $A E$ index or the more global distribution of stations used for the $D s t$ index. The $K p$ index is determined from the variability of the Earth's magnetic field, as measured by a network of ground-based magnetometers, on a 3-h basis, expressed relative to quiet-day variability on a scale from 0 to 9 . Analyzing daily averages of $K p$, we find results that are statistically consistent with those in Table 1 based on $|\mathrm{d} B / \mathrm{d} t(30 \mathrm{~m})|$; we omit that table here for brevity.

As a final test, we compare the compiled database on disturbances in the electric power grid to the catalog of solar flares maintained by NOAA, selecting only large flares of GOES classes $\mathrm{M}$ and $\mathrm{X}$ (based on the logarithmic 1-8 $\AA$ peak brightness, such that an X1 flare is 10 times brighter than an M1 flare, and close to 10 times more energetic overall (Veronig et al. 2002). For the period 1992-2010 there were 1,897 M- and X-class flares on 1,054 distinct dates. Nearly half of all M-class flares and over $90 \%$ of X-class flares are associated with CMEs (see the review by Schrijver 2009, and references therein) and thus most such flares affect the dynamics of the heliospheric field, and thereby can couple into the geomagnetic field if directed toward the Earth.

We determined the grid-disturbance frequencies $f_{a, i, r}$ using three distinct selection criteria: (1) $f_{a}$ for intervals $2-5 \mathrm{~d}$ after major solar flaring (allowing for a range of CME propagation times and a 1-2 d period of ensuing geomagnetic activity as the CME passes Earth), (2) $f_{r}$ for 4-d intervals randomly selected within $50 \mathrm{~d}$ of major solar flaring (in order to remain reasonably within similar conditions for the grid otherwise) but not within $5 \mathrm{~d}$ of that flaring, and (3) $f_{i}$ for the first 4-d intervals prior to the selected reference dates of major solar flaring that end 7-d intervals of no major solar flaring, thus selecting periods of relatively quiescent conditions in heliosphere and geospace. When selecting dates for all X- or M-class flares, Table 1 shows $f_{a}=0.151 \pm 0.006$ disturbances/day and $f_{i}=0.126 \pm 0.005$ (with the uncertainties based on the numbers of events and assuming Poisson statistics). We thus find a substantial increase in the frequency of grid disturbances in the days following major flaring relative to quiescent intervals, at a significance of about $4.5 \sigma$.

Note that $f_{r}$ is not significantly different from $f_{a}$ : with 1,054 days of $\mathrm{X}$ or $\mathrm{M}$ flaring mostly concentrated around cycle maximum (see Fig. 1), randomly selecting a date within $50 \mathrm{~d}$ from a flare frequently results in a date only days after another such major flare. When we select only days with at least one X-class flare, the chance of such overlaps is lowered: we see that in this case $f_{a}$ exceeds $f_{r}$ by about $2 \sigma$, while $f_{a}$ exceeds $f_{i}$ by $3.6 \sigma$. Dates with more than one $\mathrm{X}$ flare show an even more pronounced difference, but the sample is relatively small and the uncertainties correspondingly larger.

In order to estimate the total number $N_{\odot}$ of grid disturbances added to the background grid variability by solar activity, we multiply $f_{a}-f_{i}$ by the number of independent dates found within the set of $4-\mathrm{d}$ periods $2 \mathrm{~d}$ after major flaring, yielding $N_{\odot}=50 \pm 16$, or $4.1 \pm 1.3 \%$ of all disturbances.

The study methodology applied above enforces a strict exclusion of information bias in creating the sample and its controls by ignoring the stated reason for a power grid disturbance in the reports. This is effective in eliminating confounders related to the reporting completeness and accuracy, and allows us to quantify the impact of a single variable among all possible impacts on the US power grid, namely the grid's "exposure" to geomagnetic activity (see, e.g., Grimes \& Schulz 2005, on selection biases in samples and their controls, specifically their 
example on pp. 1429-1430). It is instructive, however, to see the impact of introducing a selection bias by a coarse separation of identified causes.

Tables 1 and 2 show the grid-disturbance frequencies when separating the disturbances into two broad categories. One category (WET) contains clear attributions to weather (including hot and cold weather, wind, ice, and lightning; 637 entries), external factors (fires, sabotage, earthquakes, collisions, etc.; 63), and technical issues (fuel shortages, maintenance, etc.; 43 entries). The complementary list (U, with 473 entries) shows causes such as "line fault", "operator error", "public appeal", "voltage reduction", "load shed", "equipment failure", etc., for which no clear correlation with weather, external, or technical issues is listed. The contrasts between $g_{a}$ and $g_{i}$ for days with geomagnetic activity in the top percentiles for the both types of events are statistically comparable to those of the full sample. The same is true for the contrast between the conditional grid-disturbance frequencies given flare activity, that is, $f_{a} / f_{i}$ for high, medium, and moderate flaring activity. We conclude from this experiment that the susceptibility of the US power grid appears to be statistically similar to geomagnetic activity for the two classes of causes, and that our findings would thus have been identical had we focused only on those disturbances for which the identified cause is clearly proximate (as follows from the examples of included causes in group $\mathrm{U}$ given above).

The results in Tables 1 and 2 are a direct demonstration of the statistically significant impact of geomagnetic activity on the US power grid. We add to that the simple visualization in Figure 2 which emphasizes this impact in a slightly different manner. As the space-weather effects on the US power grid over our 19-y interval are relatively weak, we use a superposed epoch analysis to visualize the magnitude of the effects. Figure 2 shows the average grid-disturbance frequencies for days with geomagnetic activity, as measured by $|\mathrm{d} B / \mathrm{d} t(30 \mathrm{~m})|$, in the top 2,5 , and 10 percentiles in panels $a, b$, and $c$, respectively, for 4-week periods centered on those most active dates. There clearly is a peak on the central dates relative to their surrounding periods, revealing a dependence of the US power-grid reliability on space weather conditions. Often, solar active regions exhibit series of flaring and coronal mass ejections over periods of multiple days, sometimes up to a full 2 weeks as a flare-productive region crosses the disk. Hence, for a comparison of the geomagnetically active dates with a reference date of low geomagnetic activity, the curves shown in Figure 2 do not provide suitable information to set a baseline level for grid disturbances in periods of low geomagnetic activity; that baseline level was discussed above and presented in the Tables.

In conclusion, we find a statistically significant enhancement in the frequency of power-grid disturbances on days of high geomagnetic activity, regardless of which measure for geomagnetic activity we use: a metric for 30-min variability characteristic of the central USA (for which the results from Table 1 are shown graphically in Fig. 3), a metric for the 3-h ( $K p)$ variability for high latitudes around the globe, or when looking at intervals following days of major solar flaring. This enhancement means that at least $\approx 4 \%$ of reported grid disturbances are attributable in whole or in part to enhanced geomagnetic activity. We note that although significant, the fraction of grid disturbances that we find attributable to GIC effects is relatively small, so that the overall number of disturbances attributable to space weather is small even during periods of severe solar activity: on days with the most extreme geomagnetic activity, only $\approx((0.23 \pm 0.04)-(0.06 \pm 0.02))=0.17 \pm 0.05$

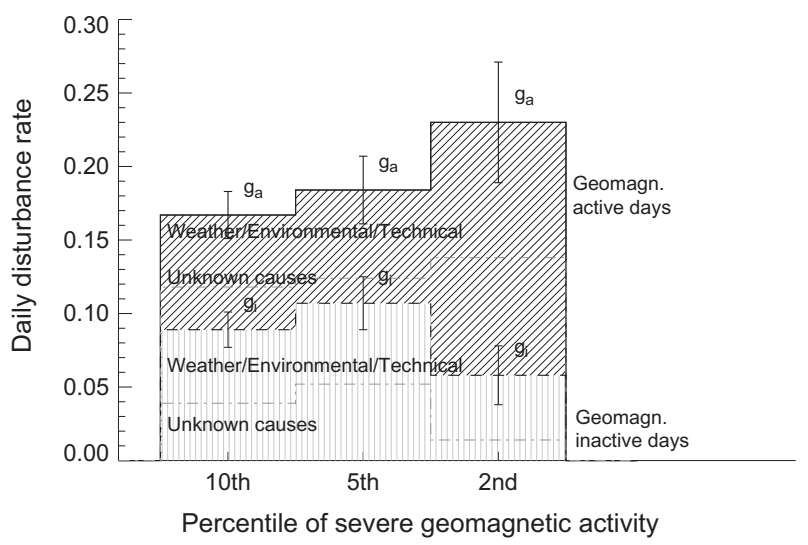

Fig. 3. Graphical rendition of the grid-disturbance rates under different levels of geomagnetic activity, as listed in Table 1. The three columns show the results for geomagnetic activity in the 10th, 5 th, and 2nd upper percentiles of geomagnetic activity as measured by $|\mathrm{d} B / \mathrm{d} t(30 \mathrm{~m})|$, respectively. The excess in event frequency on geomagnetically active days compared to nearby inactive days is shown by dark diagonal shading.

disturbances per day would be expected in association with severe space weather (using numbers from Table 1).

\section{Discussion}

We perform a retrospective cohort study to quantify the susceptibility of the US power grid to disturbances attributable (at least in part) to geomagnetic activity. The results of such a study can be expressed in a simple contingency table, of which Tables 1 and 2 are variants. Alternatively, it could be expressed as odds ratios and their confidence intervals (e.g., Morris \& Gardner 1988) or in terms of chi square, both of which can be derived from the numbers in Tables 1 and 2. Such numbers convey the same message as the combined contingency Tables 1 and 2: the impact of the "exposure" is statistically significant to more than the three-sigma level, i.e., the null hypothesis that the US power grid is insensitive to space weather is rejected with more than 0.975 (or 32 in 33 ) probability.

Except in rare cases, solar energetic events and resulting geomagnetic activity are not presently recognized as contributing to power-grid disturbances. In fact, no grid disturbance was thus attributed over the 19-y period studied, neither as primary cause nor as contributing factor, in the NERC-DOE reports. This is to be contrasted to our finding (significant in excess of 4 standard deviations) that over the 19 -y period of our study, $\approx 50$ grid disturbances reported to NERC and DOE had strong geomagnetic and solar activity at least as a contributing factor.

The present lack of recognition of geomagnetic activity as a contributing agent in grid disturbances may reflect that, in contrast to extreme storms, moderate to severe space-weather conditions do not by themselves cause such disturbances but instead are one factor among all others to which the electric power grid is susceptible. These other perturbations may be identified as the cause of the disturbance, but our study leads us to conclude that sometimes geomagnetic activity is a contributing factor. One may think of parallels such as the activity of skiers that contributes to the triggering of avalanches particularly if conditions of snowfall and weather are right; or one may consider the effect of being engaged in cell phone calls on the likelihood of vehicular accidents in demanding traffic 
conditions. We conjecture that in the grid disturbances that we find to be influenced by geomagnetic activity and their induced currents, this activity may be the equivalent of the presence of a skier or of being on the phone in the above analogies. The US power grid is, after all, a highly complex coupled system in which initially localized problems can cascade into disturbances of any size (characterized on the large end of the spectrum by a scale-free power-law distribution typical of nonlinear systems (Carreras et al. 2003; Talukdar et al. 2003), compounded by the fact that GICs induced by space weather extend over a large fraction of the footprint of the US electric power grid and thus can have effects in various locations simultaneously.

The apparent correlation of electric power grid disturbances with pronounced solar and geomagnetic activity warrants the investigation and implementation of mitigation strategies and the support of a space-weather research program as well as the continued development of a space-weather forecasting system. Such an investment would also help us to better understand what protection society would need if faced with more severe space weather than experienced in recent decades, or from more extensive cascading effects in our ever-more coupled technological infrastructure.

Acknowledgements. This work was supported by Lockheed Martin Independent Research funds. We thank D. Boteler, D. Chenette, K. Forbes, M. Hapgood, L. Lanzerotti, R. Lordan, and A. Title for discussions and the reviewers for helpful suggestions. The results presented in this paper rely on data collected at magnetic observatories. We thank the national institutes that support them and INTERMAGNET for promoting high standards of magnetic observatory practice (www.intermagnet.org).

\section{References}

Andreeova, K., T.I. Pulkkinen, M. Palmroth, and R. McPherron, Geoefficiency of solar wind discontinuities, J. Atmos. Sol. Terr. Phys., 73, 112-122, DOI: 10.1016/j.jastp.2010.03.006, 2011.

Béland, J., and K. Small, Space weather effects on power transmission systems: the cases of Hydro-Québec and Transpower New Zealand Ltd, in Effects of space weather on technological infrastructure, edited by I.A., Daglis, Kluwer Academic Publishers, The Netherlands, pp. 287-299, 2004.

Boteler, D.H., Space weather effects on power systems, in: Space Weather, Geophys. Monogr. Ser., 125, edited by P., Song, H.J. Singer, and G.L. Siscoe. AGU, Washington, DC, pp. 347-352, 2001.

Boteler, D.H., and G. Jansen van Beek, August 4, 1972 revisited: a new look at the geomagnetic disturbance that caused the L4 cable system outage, Geophys. Res. Lett., 26, 577-580, 1999.

Boteler, D.H., R.J. Pirjola, and H. Nevanlinna, The effects of geomagnetic disturbances on electrical systems at Earth's surface, Adv. Space Res., 22, 17-27, 1998.

Carreras, B., V. Lynch, D. Newman, and I. Dobson, Blackout mitigation assessment in power transmission lines, Proceedings of the 36th Hawaii International Conference on System Sciences, IEEE Computer Society, DOI: 10.1109/HICSS.2003.1173911, 2003.

FEMA, M., Managing critical disasters in the transatlantic domain the case of a geomagnetic storm. NOAA, FEMA, Washington, DC, 2010.

Forbes, K.F., and O.C. St. Cyr, Space weather and the electricity market: an initial assessment, Space Weather, 2, S10003, DOI: 10.1029/2003SW000005, 2004.
Forbes, K.F., and O.C. St. Cyr, Solar activity and economic fundamentals: evidence from 12 geographically disparate power grids, Space Weather, 6, S10003, DOI: 10.1029/2007SW000350, 2008.

Forbes, K.F., and O.C.St. Cyr, An anatomy of space weather's electricity market impact: case of the PJM power grid and the performance of its $500 \mathrm{kV}$ transformers, Space Weather, 8, S09004, DOI: 10.1029/2009SW000498, 2010.

Gaunt, C.T., and G. Coetzee, Transformer failures in regions incorrectly considered to have low GIC-risk. IEEE PowerTech, IEEE, Lausanne, 2007.

Grimes, D.A., and K.F. Schulz, Compared to what? Finding controls for case-control studies, The Lancet, 365, 1429-1433, 2005.

Hapgood, M., Lloyd's $360^{\circ}$ risk insight: Space Weather: Its impacts on Earth and the implications for business. Lloyd's, London, UK, 2011.

Hapgood, M., Prepare for the coming space weather storm, Nature, 484, 311-313, DOI: 10.1038/484311a, 2012.

JASON, Impacts of severe space weather on the electric grid (JSR11-320). The MITRE Corporation, McLean, VA, 2011.

Kappenman, J., Geomagnetic storms and their impacts on the US power grid (Tech. Rep. Meta-R-319), Metatech Corp, Goleta, CA, 2010.

Kappenman, J.G., An overview of the impulsive geomagnetic field disturbances, power grid impacts associated with the violent SunEarth connection events of 2931 October 2003 and a comparative evaluation with other contemporary storms, Space Weather, 3, DOI: 10.1029/2004SW000128, 2005.

Kappenman, J.G., L.J. Zanetti, and W.A. Radasky, Geomagnetic storm forecasts and the power industry, Eos Trans. AGU, 78, 37, 1997.

Morris, J.A., and M.J. Gardner, Calculating confidence intervals for relative risks (odds ratios) and standardized ratios and rates. British Med. J., 296, 1313-1316, 1988.

Newell, P., T. Sotirelis, K. Liou, C.I. Meng, and F.J. Rich, A nearly universal solar wind-magnetosphere coupling function inferred from 10 magnetospheric state variables, J. Geophys. Res., 112, A21026, DOI: 10.1029/2006JA012015, 2007.

PJM State and Member Training Dept, Weather and environmental emergencies. PJM, http://pjm.acrobat.com/p37769123, 2010.

Pulkkinen, T., Space weather: terrestrial perspective, Living Rev. Sol. Phys., 4, 1, 2007.

Russell, C.T., and R.L. McPherron, Semiannual variation of geomagnetic activity, J. Geophys. Res., 78, 92-108, DOI: 10.1029/JA078i001p00092, 1973.

Schrijver, C., and G. Siscoe, Heliophysics. Space storms, radiation, causes and effects, Cambridge University Press, Cambridge, UK, 2010.

Schrijver, C.J., Driving major solar flares and eruptions: a review, Adv. Space Res., 43, 739-755, DOI: 10.1016/j.asr.2008.11.004, 2009.

Schulz, K.F., and D.A. Grimes, Case-control studies research in reverse, The Lancet, 359, 431-434, 2002.

Space Studies Board, Severe space weather events - understanding societal and economic impacts. National Academy Press, Washington, DC, USA, 2008.

Talukdar, S., J. Apt, M. Ilic, L. Lave, and M. Morgan, Cascading failures: survival vs. prevention, The Electricity Journal, 16 (9), 25-31, 2003.

Veronig, A., M. Temmer, A. Hanslmeier, W. Otruba, and M. Messerotti, Temporal aspects and frequency distributions of solar soft X-ray flares, $A \& A, \mathbf{3 8 2}, 1070-1080$, DOI: 10.1051/0004-6361:20011694, 2002

Wacholder, S., D.T. Silverman, J.K. McLaughlin, and J.S. Mandel, Selection controls in case-control studies, American Journal of Epidemiology, 135, 1042-1050, 1992.

Cite this article as: Schrijver C and Mitchell S: . J. Space Weather Space Clim., 2013, 3, A19. 